\title{
Didactiser l'alternance des langues en cours de DNL
}

Jean Duverger

\section{OpenEdition}

1 Journals

Édition électronique

URL : http://journals.openedition.org/trema/302

DOI : 10.4000/trema.302

ISSN : 2107-0997

\section{Éditeur}

Faculté d'Éducation de l'université de Montpellier

\section{Édition imprimée}

Date de publication : 1 septembre 2007

Pagination : 81-88

ISSN : 1167-315X

\section{Référence électronique}

Jean Duverger, «Didactiser l'alternance des langues en cours de DNL », Tréma [En ligne], 28 | 2007,

mis en ligne le 14 janvier 2010, consulté le 30 avril 2019. URL : http://journals.openedition.org/ trema/302 ; DOI : 10.4000/trema.302

Ce document a été généré automatiquement le 30 avril 2019.

Trema 


\title{
Didactiser l'alternance des langues en cours de DNL
}

\author{
Jean Duverger
}

1 L'enseignement bi / plurilingue a plutôt bonne réputation au niveau des résultats scolaires car, d'une façon générale en effet, ceux - ci sont bons, et les élèves qui travaillent selon ce cursus sont donc considérés comme de bons élèves.

2 Alors développer ce type d'enseignement ? Pas si simple ...

$3 \mathrm{Au}$ - delà de réticences d'ordre sociologique, socio - politique ... (autre débat) la grande difficulté de leur développement est bien de pouvoir recruter des professeurs de disciplines non linguistiques (DNL), c'est - à - dire des professeurs capables d'enseigner leur discipline partiellement en langue étrangère... Enseigner les maths en anglais, la géographie en espagnol ou la biologie en allemand, pas si simple ...

Ce n'est pas simple, et ceci pour de multiples raisons :

5 - d'abord naturellement, et surtout, pour des raisons linguistiques: un professeur de sciences, d'histoire, n'est pas censé enseigner sa discipline dans une autre langue que la langue officielle de son pays... il n'est pas professeur de langue étrangère, il n'y a pas de filière de formation pour attendre un tel objectif ... (il faudrait notamment inventer des stages de formation linguistique pour les candidats / professeurs de DNL, ce n'est pas le cas),

6 - mais aussi, et on l'oublie trop, pour des raisons d'ordre didactique, parce qu'il n'y a pas de réflexion méthodologique particulière dans cette configuration pédagogique ... Comment enseigner en deux langues? Comment enseigner dans une autre langue que la langue officielle, la langue naturelle de des élèves et de la société ? Les professeurs sont démunis : pas ou peu de livres, de conseils et orientations méthodologiques...

7 - et par ailleurs, $\mathrm{a}-\mathrm{t}$ - on bien réfléchi aux impacts psychologiques et cognitifs pour l'apprenant, aux effets d'ordre sociologique, aux regards des collègues, des parents? Il 
faudrait clarifier, expliquer, rendre la société capable de comprendre et valoriser ce type d'enseignement, afin de faciliter le rôle du professeur de DNL...

Finalement, on vante les mérites, à juste titre, de l'enseignement bi / plurilingue, bénéfices d'ordres linguistiques, cognitifs, culturels, économiques, mais on ne se donne pas trop les moyens de l'étendre, de le développer, de le généraliser.

9 Cette intervention voudrait, on l'aura compris, se centrer résolument sur l'aspect didactique de l'enseignement des DNL dans le dispositif bi / plurilingue.

10 Mais d'abord soyons clair, et définissons bien ce que l'on entend ici par enseignement bi / plurilingue en général, et en cours de DNL en particulier.

11 Un dispositif bi / plurilingue n'est pas un dispositif d'immersion, il ne consiste pas à conduire des cours entièrement en langue 2 , par conséquent ...

12 Il n'est pas non plus, bien sûr, une somme de deux enseignements monolingues.

13 Il s'agit, selon nous, dans un dispositif bilingue, de conduire en DNL des unités didactiques, des cours, des leçons où les deux langues co - existent en permanence, très explicitement, où le professeur et les élèves disposent en permanence de ces deux langues, de deux outils de travail, pour le plus grand bénéfice de la discipline, au - delà bien sûr du profit pour la langue 2 (et d'ailleurs aussi pour la langue 1).

14 Il s'agit donc d'alterner les langues de manière raisonnée et efficace, de mettre en relation les messages, les méthodologies, les contenus portés par ces langues ...

15 En somme, il s'agit d'utiliser l'alternance des langues, mais en la maîtrisant, en la raisonnant, en didactisant cette alternance ...

\section{Définir des tâches, inventer des didactiques ...}

Rappelons aussi, très clairement, que le professeur de DNL est d'abord un professeur d'une discipline; le fait de l'enseigner en deux langues ne doit naturellement pas le détourner de son objectif premier qui est d' aider les enfants, par son enseignement, à s'approprier les connaissances et concepts fondamentaux de cette discipline; il doit au contraire tout mettre en œuvre pour que cette singularité de travailler en deux langues soit un "plus" pour sa discipline; il sait que cette façon de travailler améliore l'apprentissage de la langue 2 ; tant mieux ; mais il doit, me semble - $t$ - il, être au clair : ce gain linguistique n'est pour lui qu'un objectif second, il doit y avoir gain également au niveau de la construction chez les élèves des concepts de sa discipline.

Schématiquement, les pratiques actuelles du professeur de DNL peuvent se typologiser en plusieurs formules :

- Le professeur donne en L2 le cours ordinaire qu'il donnerait en L1, donc en traduisant son cours de L1 en L2 ; variantes possibles : cours en L1, puis répétitions (totales ou partielles) en L2. 
- Le professeur fait son cours ordinaire en L1 et, régulièrement, il fait un cours supplémentaire entièrement en L2, ou l'inverse ...

- Le professeur fait un cours original, articulant les contenus, les méthodologies et les langues ; on est alors dans l'enseignement "intégré », qui se décline en de nombreuses variantes : ou bien on élabore à l'avance des thématiques et des contenus originaux, ou bien, cas le plus fréquent, on prend comme trame de travail le programme officiel national et, pour chaque thématique, on fait des ajouts, des incises, des ouvertures ... trois types de tâches :

- d'abord mettre en relation, pour chaque thématique, chaque sujet d'études, des objectifs et contenus issus de manuels scolaires en L1 et en L2, en visant l'enrichissement, l'ouverture, l'approfondissement des savoirs ; si l'on garde la trame du programme officiel, on cherchera des ajouts, des incises, des références culturelles différentes, des documents qui élargissent, approfondissent, alternent les points de vue ; on est dans le comparatif, dans les regards croisés et l'on sait bien que cette approche est enrichissante. Au total, l'épistémologie de la discipline est revisitée, c'est bon pour l'enseignement - apprentissage de cette discipline.

- ensuite mettre aussi en relation des méthodologies, des stratégies d'apprentissage, des angles d'attaque différents, des conceptions différentes de l'acte d'apprendre; il y a là aussi une aide puissante aux constructions conceptuelles chez l'élève ;

- enfin, mais cette tâche est directement liée aux deux précédentes, il doit veiller à mettre en relation les langues L1et L2, en fonction des moment des documents et autres moyens utilisés, et ceci par conséquent de façon ordonnée et raisonnée, tout au long du déroulement du cours.

Mais tout cela ne s'invente pas; une formation est nécessaire pour pratiquer une alternance ainsi raisonnée.

\section{Les types d'alternance}

Je distinguerai trois types d'alternance, qui s'articulent en permanence :

- une macro - alternance, d'ordre structurel, qui concerne la programmation générale des cours, 
- une alternance séquentielle, sorte de méso - alternance, la plus délicate sans doute à maîtriser, qui se met en place tout au long de l'unité didactique,

- une micro - alternance, qui se réfère aux courts passages d'une langue à l'autre, non programmable, d'ordre très conjoncturelle par conséquent ... opérée par le professeur pendant le cours de manière raisonnée, réfléchie, volontaire, sous forme de séquences successives et ceci, dans la perspective de favoriser chez les élèves la mise en œuvre des processus d' apprentissage. peut prendre par conséquent différentes configurations, a pour objet d'enrichir les contenus, de croiser les documents en différentes langues, de varier les entrées 
méthodologiques; en stimulant les comportements de concentration, de curiosité, d'attention, de mémorisation, de flexibilité cognitive en général, cette méso - alternance peut permettre en effet de faciliter les constructions conceptuelles disciplinaires.

Proposons ici quelques pratiques pédagogiques, recueillies tout au long de stages de formation réunissant des professeurs de DNL de plusieurs pays européens, des stages visant à améliorer l'efficacité des enseignements de DNL, (en favorisant par conséquent les apprentissages) sans pour autant prendre du retard dans les programmes (souci permanent et légitime des professeurs).

On donnera donc ici synthétiquement quelques repères identifiés, en suivant le déroulement habituel d'une unité didactique.

- Le titre de "l'unité didactique » doit être donné en L1 et L2 ; c'est là tout à fait important pour des raisons d'abord symboliques (c'est un signe fort qui indique que la « leçon », le « cours » va se dérouler en deux langues) mais aussi parce que, souvent, les deux intitulés du titre peuvent être assez éloignés d'une traduction littérale... et c'est déjà un motif de réflexion ...

- Le premier moment d'une " unité didactique " est typiquement un moment "d'émergence des représentations ", un moment où l'on fait le point, en grand groupe, mais aussi individuellement, sur ce que l'on sait ou croit savoir à propos de la thématique visée... Cette phase doit impérativement se dérouler en L1, puisque c'est en L1 qu'ont été vécues et que se sont construites ces représentations, images et idées souvent « préconçues » qui vont devoir, le plus souvent, être remises en cause lors du déroulement de l'unité didactique ...

- Le travail central qui expose et traite la thématique dans la forme la plus pertinente par rapport au thème traité vient ensuite, et c'est là que textes et documents en L1 et L2 peuvent alterner, se croiser, se mettre en relation ...

De la même façon, au delà des textes, une alternance est souhaitable entre les deux langues en ce qui concerne les graphiques, cartes, schémas et autres statistiques.

Si le travail nécessite des observations, des expérimentations, des mesures, on tâchera aussi de les conduire dans l'une ou l'autre langue, mais sans souci naturellement de « comptabiliser » quoi que ce soit, d'équilibrer ou de respecter des parités illusoires.

L'idée centrale est ici d'avoir le souci de chercher entre L1 et L2 des complémentarités, des précisions, des ouvertures, des entrées différentes susceptibles d'aider aux apprentissages.

- Un point particulier mérite d'être souligné : en encadré, on pourra fabriquer un double lexique (en $L 1$ et $L 2)$ des termes spécifiques relatifs au sujet traité, sorte de répertoire de mots clés.

- Les prises de notes, les mesures ou observations seront formulées indifféremment en L1 ou L2.

- Les conclusions intermédiaires, résumés et synthèses finales, théorèmes, lois, règles, axiomes etc. seront obligatoirement formulés dans les deux langues, à l'oral et à l'écrit, en s'aidant de manière systématique des manuels scolaires utilisés en L1 et L2 : les formes langagières utilisées dans les deux langues sont en effet souvent différentes, loin des traductions littérales et il est probablement utile de travailler et retenir les deux formulations, afin de favoriser la mémorisation et la conceptualisation.

- Les cahiers, classeurs, ou autres outils de travail utilisés par les élèves pour " apprendre ", devraient être rédigés et illustrés dans les deux langues, surtout naturellement s'ils incluent les synthèses et conclusions ci - dessus évoquées. 
- Les exercices de contrôle et l'évaluation.

- Il est important de proposer des exercices de contrôle et d'évaluation dans les deux langues. Les formes peuvent naturellement considérablement varier selon les exercices, mais cette utilisation des deux langues à ce niveau est fondamentale si l'on veut tirer un bénéfice optimal de l'enseignement bilingue.

Ainsi les problèmes, les tests, les QCM, les questions - réponses ... les exercices de toutes sortes seront posées dans une langue ou l'autre ; de nombreuses configurations sont possibles ... (on peut par exemple poser une question dans une langue et demander la réponse dans l'autre).

D'une manière générale, les évaluations intermédiaires ou terminales (y compris les certifications des dernières années de scolarité) devraient être mises en place en utilisant les deux langues d'apprentissage. amélioration des constructions conceptuelles dans le dispositif d'enseignement bilingue.

- Est - il aberrant de penser par exemple que la conceptualisation et l'abstraction sont plus aisées avec une langue abstraite, non affective, qu'avec la langue naturelle, maternelle, très chargée d'affectivité ; donc plus aisée avec L2 qu'avec L1?

- Peut - on raisonnablement penser que , pour la même raison, le traitement et l'abandon des représentations premières, voire des « fantasmes scientifiques » est plus facile avec L2 qu'avec L1?

- Est - il anormal de penser que le fait de travailler un concept selon d'autres entrées méthodologiques, en utilisant d'autres types de documents, en reformulant et synthétisant d'une autre façon, grâce à l'autre langue, de penser par conséquent que tout cela favorise les constructions conceptuelles en introduisant une pédagogie comparatiste, une pédagogie de l'écart... ?

- Peut - on penser que de disposer de deux outils langagiers est plus fécond que d'en utiliser un seul : deux outils (deux marteaux, deux pinces, deux tournevis... pour clouter, visser etc.) n'est - ce pas utile?

- Peut - on penser aussi que les conditions de l'apprentissage sont, dans une certaine mesure, facilitées lorsqu'une langue 2 est utilisée, parce que celle - ci nécessite, pour être comprise, plus d'attention, de concentration, de mémorisation que la langue naturelle?

- Et d'autres facteurs interviennent sans doute : on travaille davantage dans ces sections car il y a souvent quelques heures de cours en plus, la classe devient souvent une " bonne classe ", les professeurs s'investissent alors davantage, une certaine émulation se développe etc., etc. (ce qui ne manque pas de poser quelquefois des « problèmes » au sein des établissements scolaires ...).

Toutes ces hypothèses interférent sans doute et mériteraient en tous cas d'être creusées ; quoiqu'il en soit, les sections bi / plurilingues fonctionnent globalement de manière satisfaisante, une bonne évaluation naturelle étant d'observer que les départs de ces classes sont fort rares, que les résultats aux évaluations et certifications sont bons ou très bons, et que les demandes d'intégration sont nombreuses ...

\section{Quelques propos conclusifs}

Dans l'enseignement bilingue tel que nous l'avons défini, quels que soient les programmes et contenus, les deux langues co - existent, se côtoient, alternent au quotidien. C'est un aspect méthodologique important, c'est une réalité, c'est une chance qu'il est capital et 
bénéfique d'exploiter. Curieusement, au cours de stages et rencontres, lorsqu'on interroge les enseignants de DNL concernés à propos de la manière dont ils travaillent dans les deux langues, les réponses sont souvent évasives et embarrassées, comme si la question était un peu dérangeante; et on se rend vite compte, en fait, que d'un pays à l'autre, d'un établissement à l'autre, chacun fait comme il peut, de manière quelque peu empirique. Sur ces points, et indépendamment des directives pédagogiques (lorsqu'elles existent) dans ces sections bilingues, les représentations sont prégnantes et font plutôt pencher les opinions et points de vue vers la logique de l'immersion ponctuelle, du discours monolingue tout au long d'une même séquence. On peut d'ailleurs relever chez certains enseignants qui alternent les langues de vagues sentiments de culpabilité, sans doute parce que, comme l'écrivait JANKÉLÉVITCH, «toutes conditions étant égales, l'incomposé vaut mieux que le composé; sur ce préjugé reposent la prohibition des mélanges, la phobie des croisements, l'horreur du métissage qui forment le fond d'un purisme simpliste ».

De telle façon que lorsqu'on observe de près les programmations de ces sections bilingues, lorsqu'on entre dans les classes, on a souvent du mal à percevoir des logiques d'alternance, à comprendre les raisons des choix de langues.

La littérature pédagogique n'est effectivement pas très abondante sur ces points et il ne se dégage guère de consensus autour de cette question.

C'est probablement en Vallée d'Aoste où l'enseignement bilingue est institutionnalisé et généralisé, que la réflexion didactique concernant l'alternance a été la plus poussée, grâce aux enseignants et aux chercheurs - didacticiens qui les ont accompagnés.

Didactiser l'alternance ... ça n'est pas la tradition pédagogique la plus courante, c'est même encore un peu mal vu, pour ne pas dire plus ...

Ces quelques propos n'avaient pour ambition que de proposer un peu d'ordre ... Mais attention, comme le disait Paul VALÉRY, deux dangers menacent le monde : « l'ordre et le désordre » ...

\section{BIBLIOGRAPHIE}

CASTELLOTTI, V. (1997), « Langue étrangère et français en milieu scolaire : Didactiser l'alternance », in Alternances des langues et apprentissages, pp. 401 - 410, éla, n 108. Paris : Didier Erudition.

CAUSA, M. (2007), «L'indispensable alternance codique », in Le français dans le monde, $\mathrm{n}^{\circ} 351$. Paris : CLE International.

CAVALLI, M. (2006). Éducation bilingue et plurilinguisme. Coll. LAL. Paris : Didier.

COSTE, D., (1997), « Alternances didactiques », pp. 393 - 400, in Etudes de Linguistique Appliquée, n 108. Paris : Didier Erudition.

DUVERGER, J. (2003). « Enseigner en français, pas si simple », in Le français dans le monde, $\mathrm{n}^{\circ} 329$. Paris : CLE International.

DUVERGER, J. (2005). L'enseignement en classe bilingue, Paris : Hachette. 
FLE. DUVERGER, J (2007) « Professeur bilingue de DNL, un nouveau métier », in Le français dans le monde, $\mathrm{n}^{\circ} 349$. Paris : CLE International.

GAJO, L. (2006) « L'enseignant en classe bilingue, un nouvel enseignant ? », in Le français dans le monde, $\mathrm{n}^{\circ} 347$. Paris : CLE International.

JANKÉLÉVITCH, V. (1960), Le pur et l'impur. Paris : Flammarion.

SIMON, D. (1997). Alternance codique en classe de langue : rupture de contrat ou survie? Alternances des langues et apprentissages, éla ${ }^{\circ} 108$. Paris : Didier Erudition.

\section{RÉSUMÉS}

Dans l'enseignement bilingue, il semble naturel et de bon aloi d'admettre que les cours de disciplines non linguistiques (DNL) soient conduits tantôt en L1, tantôt en L2 ; cette idée simple (simpliste) ne semble ni réaliste (on observe que les deux langues sont souvent sollicitées au cours d'une même séquence) mais surtout ni pertinente, ni efficace; la didactisation de l'alternance des langues pendant le déroulement des unités didactiques favorise largement les constructions conceptuelles disciplinaires, sans préjudice des bénéfices linguistiques.

With regard to bilingual teaching, it is generally held as normal and correct that classes of non linguistic subjects be taught alternatively in L1 and in L2. However, this simple (simplistic) idea does not appear realistic (both languages are often used within a single pedagogical sequence) and, moreover, seems neither relevant nor efficient. The didactic integration of language shifting within a single teaching unit is a facilitating factor for concept and knowledge building in the subject, not mentioning linguistic benefits.

\section{INDEX}

Mots-clés : alternance codique, didactique, DNL, enseignement bilingue

Keywords : bilingual teaching, code shifting, didactics

\section{AUTEUR}

\section{JEAN DUVERGER}

Inspecteur de l'éducation honoraire, Président de l'Association pour le développement de l'enseignement bi / plurilingue 